\title{
Interference Information Based Power Control for Cognitive Radio with Multi-Hop Cooperative Sensing
}

\author{
Youngjin YU ${ }^{\dagger a)}$, Student Member, Hidekazu MURATA ${ }^{\dagger}$, Koji YAMAMOTO $^{\dagger}$, Members , \\ and Susumu YOSHIDA ${ }^{\dagger}$, Fellow
}

\begin{abstract}
SUMMARY Reliable detection of other radio systems is crucial for systems that share the same frequency band. In wireless communication channels, there is uncertainty in the received signal level due to multipath fading and shadowing. Cooperative sensing techniques in which radio stations share their sensing information can improve the detection probability of other systems. In this paper, a new cooperative sensing scheme that reduces the false detection probability while maintaining the outage probability of other systems is investigated. In the proposed system, sensing information is collected using multi-hop transmission from all sensing stations that detect other systems, and transmission decisions are based on the received sensing information. The proposed system also controls the transmit power based on the received CINRs from the sensing stations. Simulation results reveal that the proposed system can reduce the outage probability of other systems, or improve its link success probability.

key words: cognitive radio, cooperative sensing, shadowing, interference information, multi-hop transmission
\end{abstract}

\section{Introduction}

Recently, the demand for wireless communications has increased dramatically. As the demand for wireless communications is increasing, frequency band that has good propagation characteristics is becoming scarce. A cognitive radio technique that enables sharing of the frequency band by multiple systems may be the answer to this problem [1]-[3]. In this paper, two wireless systems sharing the same frequency band are considered. A short-range wireless system attempts to use this frequency band without excessive interference to other systems. Thus, it is crucial to accurately detect other systems. However, in wireless communication channels, multipath fading and shadowing cause uncertainty in the received signal level. As a result, individual sensing is not adequate to detect other systems with high accuracy.

Cooperative sensing techniques in which the sensing results are shared among the stations can improve the detection probability of other systems [4], [5]. In [6], the sensing information is weighted by the distance from the sensing station. Cooperative sensing can detect another system robustly in severe fading environments. Moreover, cooperative sensing can reduce the detection time and thus increase the overall agility of the system [7].

In spatial correlated shadowing environments, however, we cannot detect other systems robustly, even if co-

Manuscript received May 7, 2007.

Manuscript revised July 20, 2007.

${ }^{\dagger}$ The authors are with the Graduate School of Informatics, Kyoto University, Kyoto-shi, 606-8501 Japan.

a) E-mail: contact-h19@hanase.kuee.kyoto-u.ac.jp DOI: 10.1093/ietcom/e91-b.1.70 operative sensing is employed [4]. In this case, it is more effective to select a few independent sensing stations (that are distant from each other) than to select several correlated sensing stations. In order to receive independent sensing information, multi-hop transmission can be employed in cooperative sensing when the sensing information is exchanged. However, sensing information reported by several sensing stations may increase the false detection probability. The most important consideration is how to prevent another system from being interfered with while reducing the false detection probability. In this paper, a system collecting not only sensing information but also interference information through multi-hop transmission is proposed.

The proposed system receives sensing information using multi-hop transmission from all sensing stations that detect other systems. The transmitter determines whether to transmit based on the received sensing information. Moreover, the transmitter adjusts its transmit power based on the interference information received from the sensing stations. By receiving the interference information together with the sensing information, the proposed system can estimate the impact of its transmission to another system. In this paper, CINR values are used as the interference information. The proposed system is evaluated in terms of the outage probability and the link success probability. It is shown that the proposed system can reduce the outage probability of other systems, or improve its link success probability.

The remainder of this paper is organized as follows. In Sect. 2, the system model and the investigated sensing schemes are described. Section 3 presents the simulation conditions and the simulation results along with discussions. Finally, Sect. 4 presents the conclusions.

\section{System Model and Sensing Schemes}

Assume that there are two systems that share the frequency band. As a high-priority system (system A), we consider a relatively long-range wireless system. There are one transmitter and many receivers in the system A. The low-priority system (system B) is assumed to be a short-range multi-hop system. The system B detects the system A and reuses the shared frequency band spatially.

It is assumed that the system B has no position information. Instead, we exploit the received signal level information. Moreover, we assumed that the system A is a noncognitive system. Thus, the system B cannot receive any 
information from the system A directly.

In order to avoid interference with the system $\mathrm{A}$, the transmitter of the system B (SB-Tx) does not start transmission to the receiver of the system $B$ (SB-Rx) when the transmitter of the system A (SA-Tx) is detected by the system B. If the received signal level of the SA-Tx exceeds the signal sense threshold $T_{\text {detect }}$, we consider that the system B detects the SA-Tx.

There are multipath fading and shadowing that cause uncertainty in the received signal level. If the system B does not detect the system A, the receiver of the system A (SA$\mathrm{Rx}$ ) may be interfered by the SB-Tx. Since there is a tradeoff between the outage probability of the system A and the link success probability of the system B, the link success probability is evaluated by fixing the maximum value of the outage probability of the system A. The received signal level varies with not only multipath fading and shadowing but also distance. We are interested in reusing the shared frequency band spatially, so the performance is evaluated by changing the distance $d$ between the SA-Tx and the SB-Tx.

\subsection{Individual Sensing}

As the simplest sensing scheme, we consider individual sensing. In individual sensing, the detection of the SA-Tx is performed by the SB-Tx only. However, as a result of the multipath fading and shadowing, individual sensing is not adequate to detect the system A with high accuracy.

\subsection{Cooperative Sensing Making Use of Multi-Hop Trans- mission}

In cooperative sensing, the more stations that are involved in sensing, the higher the sensitivity of detection. The reason for this is that the system B can detect the system A even if only one station detects the SA-Tx. However, in correlated shadowing, it is more effective to employ spatially independent stations [4]. In order to receive sensing information over a wide area, multi-hop transmission is employed in cooperative sensing.

In the system B, there are three types of stations: sensing stations (SB-SS), a data transmission station (SB-Tx), and a data reception station (SB-Rx). In our system model, there is one SB-Tx and one SB-Rx in the system B. To simplify the analysis, the SB-Tx communicates with the SB-Rx in single-hop transmission.

Sensing information is exchanged through multi-hop transmission in the system B, as shown in Fig. 1. Suppose a SB-SS ${ }_{j}$ receives the signal from the SA-Tx having the received signal level of $S_{\mathrm{A}, j}$. If $S_{\mathrm{A}, j}$ is larger than $T_{\text {detect }}$, then the $\mathrm{SB}_{-S S_{j}}$ transmit their station ID $j$ and received signal level of the SA-Tx $S_{\mathrm{A}, j}$. Each SB-SS ${ }_{j}$ periodically measures $S_{\mathrm{A}, j}$, and shares the information among the system B stations.

The SB-Tx receives the sensing station ID $j, S_{\mathrm{A}, j}$ and the number of hops $N_{\text {hop }, j}$ from the SB-SS ${ }_{j}$. As the system $\mathrm{B}$ has no position information, the transmission of the SB-

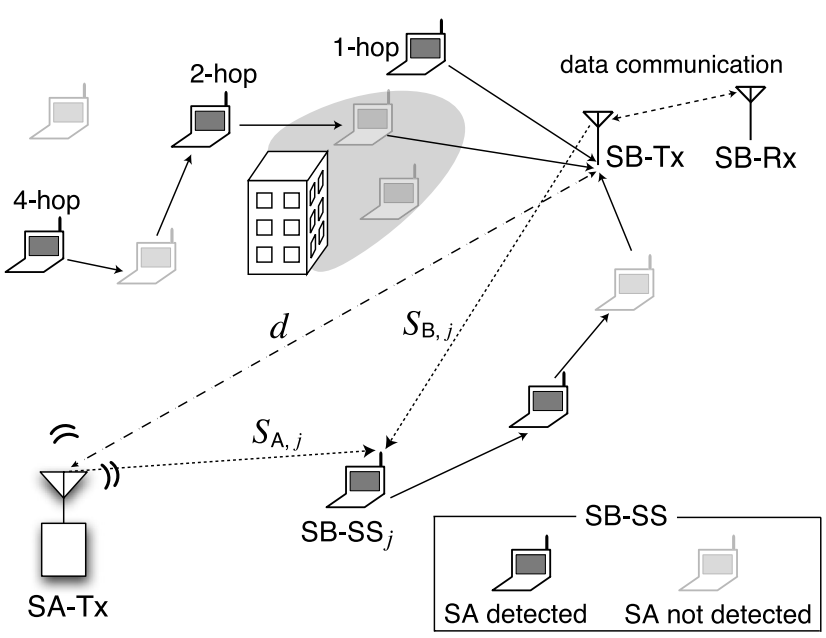

Fig. 1 Exchange of system A sensing information in system B.

Tx is determined by the minimum number of hops. When none of the SB-SS ${ }_{j}$ of $N_{\text {hop } j}=1$ do detect the SA-Tx, it is assumed that the SB-Tx is sufficiently far from the system A and would not interfere with the system A. Therefore, when the minimum number of hops among all of the received sensing information exceeds or equals two, the SBTx starts transmission.

In this paper, a routing that minimizes $N_{\text {hop }, j}$ is assumed in the system B. The system B is assumed to have a control channel for multi-hop transmission, and the sensing information is exchanged through the control channel. The detailed routing algorithm and the required bandwidth for this control channel are beyond the scope of this paper.

\subsection{Cooperative Sensing with CINR Report}

We propose a new cooperative sensing scheme that receives the CINR information and adjusts its transmit power. In the cooperative sensing with CINR report (CSCINR), the transmission of the SB-Tx is also determined based on the minimum value of $N_{\text {hop }}$.

Because we assume that the system B cannot receive any information from the system A, the CINR information of the system A is estimated by the system B. The SB-SS also measures $S_{\mathrm{A}, j}$, the received signal level of the SB-Tx $S_{\mathrm{B}, j}$, and the received noise level $N$, and then three values are reported to the SB-Tx as a CINR information.

However, the location of the SB-SS is different from that of the SA-Rx, and there is uncertainty in the received signal level caused by multipath fading and shadowing. As a result, the signal level of the SA-Tx and the SB-Tx in the SA-Rx are different from $S_{\mathrm{A}, j}$ and $S_{\mathrm{B}, j}$ of the SB-SS ${ }_{j}$. Therefore, the CINR information should be reported from all of the SB-SS, and the smallest CINR value is employed in the transmit power control.

In order to keep the CINR information traffic reasonable, a CINR reporting condition is employed. If $S_{\mathrm{A}, j}$ of the SB-SS $S_{j}$ is between $T_{\text {detect }}$ and $2 T_{\text {detect }}$, then the SB-SS ${ }_{j}$ reports the CINR information to the SB-Tx. If a wider CINR 
reporting condition is employed, greater interference reduction will be possible. However, under the assumption of this paper, the above reporting condition can receive sufficient CINR information, and a wider range did not yield a further reduction in interference.

From the received CINR information, the SB-Tx can estimate the current interference conditions toward the system $\mathrm{A}$. When the received CINR information is smaller than the required CINR $\gamma_{\text {req }}$, the SB-Tx adjusts its transmit power so that the CINR of the SB-SS is satisfied. Here, we consider the following transmit power control. We decide the new system B transmit power $P_{\mathrm{SB} \text {,new }}$ based on the smallest CINR $\gamma_{M}$ among all received CINRs and the current transmit power $P_{\mathrm{SB}, \text { now }}$. Here, $M$ is defined as follows:

$$
M=\arg \min _{j} \frac{S_{\mathrm{A}, j}}{S_{\mathrm{B}, j}+N} .
$$

The parameters of this algorithm are assumed as follows,

- Permissible interference level of the system A is $I_{\mathrm{SA}}$.

- Interference level of the SB-Tx at SB-SS ${ }_{M}$ is $S_{\mathrm{B}, M}$.

- Received signal level of the SA-Tx at $\mathrm{SB}_{-} \mathrm{SS}_{M}$ is $S_{\mathrm{A}, M}$.

- Noise level is $N$.

We assume that the smallest CINR $\gamma_{M}$ is $x$ times lower than the required CINR $\gamma_{\text {req. }}$. Therefore, we can obtain $\gamma_{\text {req }}$ by multiplying $\gamma_{M}$ and $x$.

$$
\begin{aligned}
& \gamma_{M} x=\gamma_{\mathrm{req}} \\
& \frac{S_{\mathrm{A}, M} x}{S_{\mathrm{B}, M}+N}=\frac{S_{\mathrm{A}, M}}{I_{\mathrm{SA}}+N} .
\end{aligned}
$$

From this equation we can derive $P_{\mathrm{SB}, \text { new }}$ as follows,

$$
\begin{aligned}
P_{\mathrm{SB}, \text { new }} & =P_{\mathrm{SB}, \text { now }} \frac{I_{\mathrm{SA}}}{S_{\mathrm{B}, M}} \\
& =\frac{P_{\mathrm{SB}, \text { now }}\left(S_{\mathrm{B}, M}+(1-x) N\right)}{x S_{\mathrm{B}, M}} .
\end{aligned}
$$

The transmit power of SB-Tx is adjusted using Eq. (3) so that the interference level of SB-Tx at SB-SS ${ }_{M}$ becomes $I_{\mathrm{SA}}$.

The CSCINR, which is described above, would interfere with the system A until the CINR information from the SB-SS is received. However, if the SB-Tx increases the transmit power in small steps from the transmit power which does not interfere with the system A, the CSCINR can avoid interference with the system A. Thus, we do not consider the interference to the system A until the CINR information is received.

\section{Performance Evaluations}

Three sensing schemes, individual sensing (IS), cooperative sensing (CS), and cooperative sensing with CINR report (CSCINR), are analyzed in terms of the outage probability and the link success probability. First, we show that the CSCINR can reduce the interference to the system A more than the CS when the detection probability is the same in the CS and the CSCINR. In addition, we show that the
CSCINR can improve the link success probability of the system B when the outage probability of the system A is the same among three sensing schemes. Most of the research [4]-[6] on the cooperative sensing has focused on the detection probability of the system A and little attention has been given to the link success probability of the system B. The investigation of the link success probability of the system B is essential in order to evaluate the sensing schemes.

\subsection{Simulation Conditions}

The simulation parameters are shown in Table 1, and the simulation model is illustrated in Fig. 2. We assume a $10 \mathrm{~km}$ square area. The SA-Tx is located at $(300 \mathrm{~m}, 300 \mathrm{~m})$, and the $\mathrm{SA}-\mathrm{Rx}$ is located at the edge of the system A service area, where the received signal level from the SA-Tx becomes $-94 \mathrm{dBm}$ by path-loss only. The SB-Rx is located $100 \mathrm{~m}$ from the SB-Tx in a random direction, and in the simulations, 500 different positions having the same distance to the SB-Tx are examined. The SB-SS is uniformly distributed in the simulation area.

The interference from the SB-Tx is evaluated by the ratio of interfered SA-Rx $\rho$. We consider the SA-Rx to be interfered with when the CINR value of the SA-Rx does not exceed $\gamma_{\text {req. }}$. We count, in advance, the number of SA-Rx having CINRs that do not exceed $\alpha \gamma_{\text {req }}$ due to shadowing,

Table 1 Parameters used in the simulation.

\begin{tabular}{l|r}
\hline Parameters & Values \\
\hline Number of system B sensing stations & 85,000 \\
Carrier frequency & $1.9 \mathrm{GHz}$ \\
Bandwidth & $288 \mathrm{kHz}$ \\
Required CINR $\gamma_{\text {req }}$ & $15 \mathrm{~dB}$ \\
Noise figure & $10 \mathrm{~dB}$ \\
Antenna gain & $0 \mathrm{dBi}$ \\
Path loss exponent & 3.0 \\
Transmit power of system A & $35 \mathrm{dBm}$ \\
Transmit power of system B & $12.7 \mathrm{dBm}$ \\
\hline
\end{tabular}

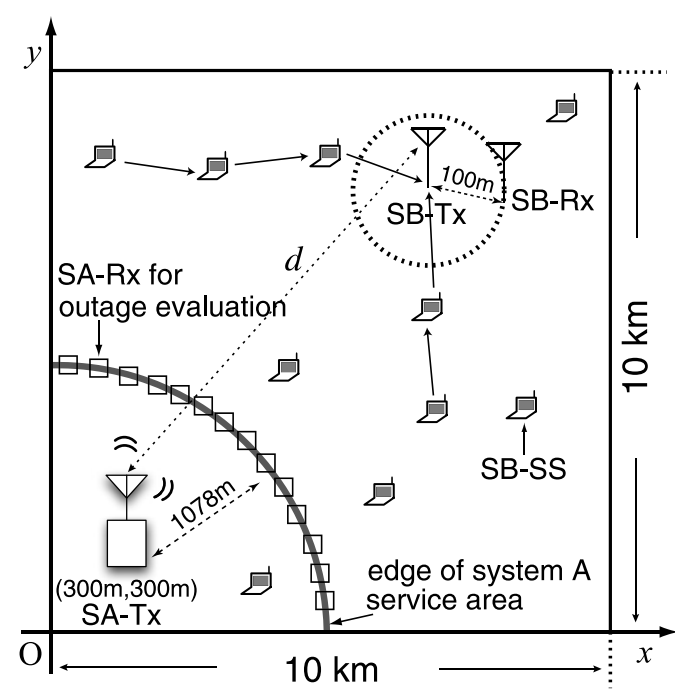

Fig. 2 Simulation model. 
and the increase in this number due to the transmission of the SB-Tx is assumed to be the number of interfered SA$\mathrm{Rx}$ from the SB-Tx. $\alpha$ is the margin in the received signal level. In this paper, $\alpha=0 \mathrm{~dB}$ and $0.5 \mathrm{~dB}$ are investigated. The maximum value of the interfered SA-Rx $\rho_{\max }$ is controlled by changing $T_{\mathrm{IS}}, T_{\mathrm{CS}}$, and $T_{\mathrm{CSCINR}}$. The $T_{\mathrm{IS}}$, $T_{\mathrm{CS}}$, and $T_{\mathrm{CSCINR}}$ is the signal sense threshold of IS, CS, and CSCINR, respectively.

Since the SB-Tx can start the transmission when the SA-Tx is not detected, the link success probability of the system B $p_{\text {link }}$ is calculated by multiplying the transmission probability $p_{\mathrm{tx}}$ with the link success probability for each transmission $p_{\text {one }}$. The probability of starting the transmission is $p_{\mathrm{tx}}$. The link success probability for each transmission $p_{\text {one }}$ is evaluated by the CINR of the SB-Rx. When the CINR of the SB-Rx exceeds $\gamma_{\text {req }}$, the communication of the system B succeeds. The averaged value is $p_{\text {one }}$ in that distance.

Since the signal is measured over a comparatively long time, the multipath fading is assumed to be averaged out and so was not considered. Shadowing is assumed to be the log-normal distribution of standard deviation $6 \mathrm{~dB}$ and the shadowing values are assumed to be the same for all of the schemes.

In this paper, the system B is assumed to have a dedicated control channel for the sensing and interference information exchange. In this control channel, multihop transmission is assumed to be free from interference. As a result, the link success of the multi-hop transmission is determined by the transmit power, noise level, shadowing value, and distance. We have the maximum number of hops 5 to be relayed in order to limit the amount of received information at the SB-Tx.

\subsection{Reduction of Interference to System A}

In this section, we investigate the interference reduction effect of the CSCINR by assuming the same signal sense threshold in the CS and the CSCINR. In order to make the $\rho_{\max }$ of IS and CS become $1 \%, T_{\mathrm{IS}}$ and $T_{\mathrm{CS}}$ are set to $-111.3 \mathrm{dBm}$ and $-93.2 \mathrm{dBm}$, respectively. The transmission is determined by the sensing information from the $\mathrm{SB}_{-} \mathrm{SS}_{j}$ of $N_{\text {hop }, j}=1$ in the CS and the CSCINR. Moreover, $T_{\mathrm{CSCINR}}$ is equals to $T_{\mathrm{CS}}=-93.2 \mathrm{dBm}$. In addition, $\alpha=0 \mathrm{~dB}$ is assumed.

The ratios of the SA-Rx that interfered with the SBTx are shown in Fig. 3. From this figure, we can know that when the signal sense threshold is the same in the CS and the CSCINR, the CSCINR can reduce the $\rho_{\max }$ to less than $0.4 \%$ by adjusting its transmit power based on the CINR information. Although the transmit power is adjusted to reduce the interference, there are $0.4 \%$ interfered SA-Rx. This is because the shadowing values are different for the $\mathrm{SA}-\mathrm{Rx}$ and the $\mathrm{SB}-\mathrm{SS}_{M}$. If the shadowing loss from the SB-Tx to the SA-Rx is smaller than that to the SB-SS ${ }_{M}$, the SA-Rx is interfered by the SB-Tx, even though the transmit power is adjusted.

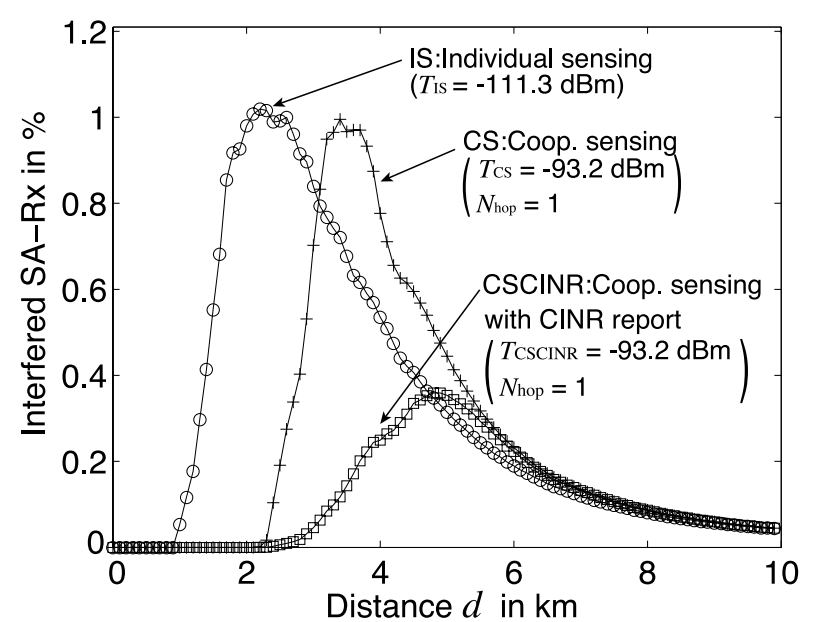

Fig. 3 Ratio of system A receivers interfered with by SB-Tx. $\rho_{\max }$ of IS and $\mathrm{CS}$ is fixed to $1 \%$.

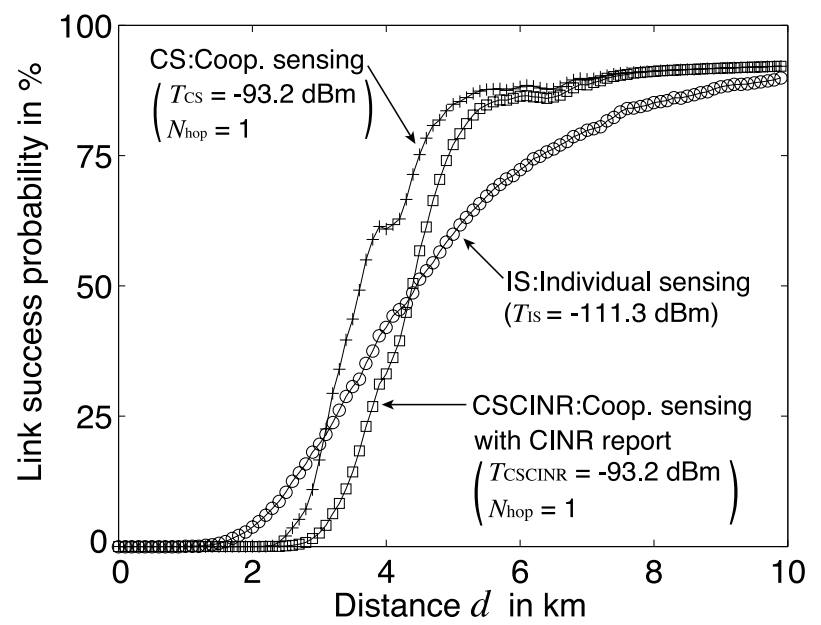

Fig. 4 Link success probability of system B $p_{\text {link }} . \rho_{\max }$ of IS and CS is fixed to $1 \%$.

As shown in Fig. 4, the $p_{\text {link }}$ of the IS is lower than the other schemes, and the $p_{\text {link }}$ of the CS increases greatly from around $3 \mathrm{~km}$. On the other hand, $p_{\text {link }}$ of the CSCINR is even lower than the IS in the vicinity of the SA-Tx. This is the trade-off between interference and link success. As the SB-Tx becomes far from the SA-Tx, $p_{\text {link }}$ of the CSCINR reaches the same value of the CS.

In order to analyze the difference of $p_{\text {link }}$ as shown in Fig. 4, we investigate $p_{\mathrm{tx}}$ and $p_{\text {one }}$, as shown in Figs. 5 and 6 , respectively. In order to be robust for rare events, the IS sets low $T_{\mathrm{IS}}=-111.3 \mathrm{dBm}$. As a result, the $p_{\mathrm{tx}}$ of the IS is lower than that of the other schemes. Since the CS and the CSCINR have the same detection probability, $p_{\mathrm{tx}}$ of the CSCINR is equal to that of the CS.

Figure 6 shows the $p_{\text {one }}$ of the three sensing schemes. Since the noise level and the shadowing value is the same in the three schemes, $p_{\text {one }}$ is determined by the transmit power of the SB-Tx and $d$. Since the transmit power of the IS is the same as that of the CS, $p_{\text {one }}$ of the IS is the same as that of the CS for the same $d$. However, since the CSCINR adjusts 


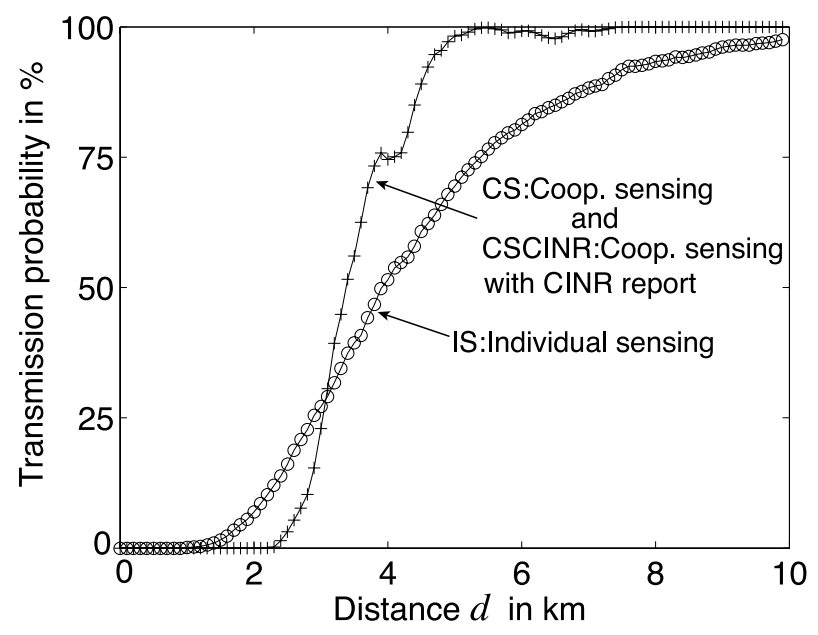

Fig. 5 Probability of starting SB-Tx transmission $p_{\mathrm{tx}}$ when there is a request for transmission.

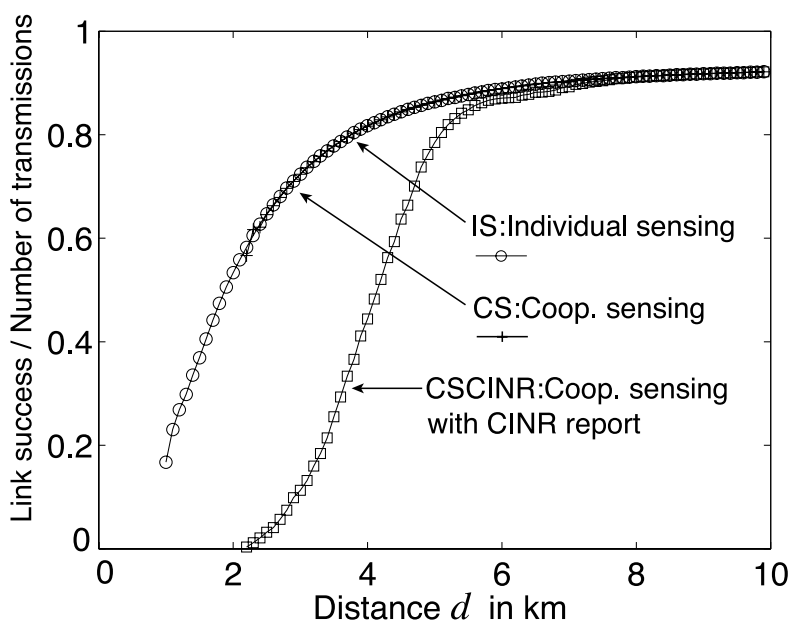

Fig. 6 Link success probability for each transmission of SB-Tx $p_{\text {one }}$.

its transmit power, $p_{\text {one }}$ of the CSCINR is lower than that of the other schemes.

The difference in $p_{\text {link }}$ between the IS and the CS, as shown in Fig 4, is caused by the difference of $p_{\mathrm{tx}}$. Similarly, the difference in $p_{\text {link }}$ between the CS and the CSCINR is caused by the difference of $p_{\text {one }}$.

\subsection{CINR Distribution of Interfered System A Receivers}

We investigate the CINR distribution of the interfered SA$\mathrm{Rx}$ when there is no interference from the SB-Tx. Figure 7 shows the results at $d=3.5 \mathrm{~km}$. Since we assume that the shadowing value and the received signal level of the SA$\mathrm{Rx}$ are the same among the three schemes, the CINR distribution is determined by the transmit power of the SB-Tx. Since the transmit power of the IS and the CS are the same, the CINR distribution is the same. However, the CSCINR adjusts its transmit power so that the CINR distribution is different. The interfered SA-Rx in the CSCINR has, before the transmission of SB-Tx, a low CINR ranging from $15 \mathrm{~dB}$

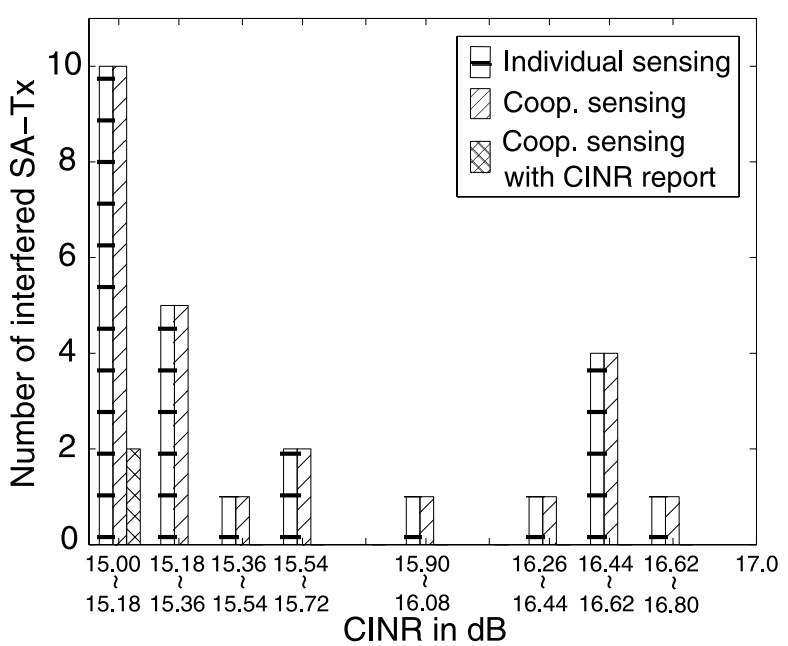

Fig. 7 CINR distribution of interfered system A receivers by SB-Tx. Required CINR is $15 \mathrm{~dB}$.

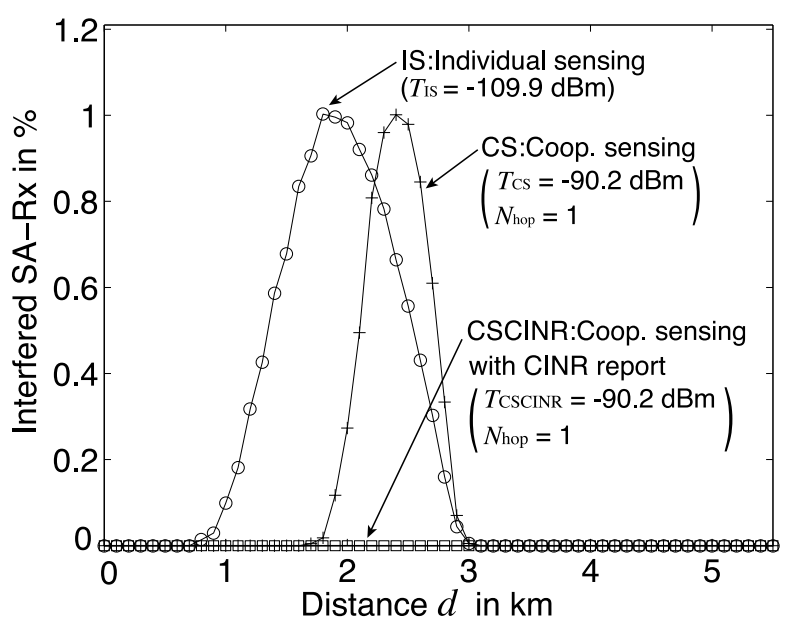

Fig. 8 Ratio of system A receivers interfered with by SB-Tx. The receiver of system A has a margin of $0.5 \mathrm{~dB}$ or more in the received signal level.

to $15.18 \mathrm{~dB}$, as shown in Fig. 7. These SA-Rx are interfered with because the CINR becomes lower than the threshold of $15 \mathrm{~dB}$ after the SB-Tx starts its transmission.

We are interested in how the performance changes when $\alpha=0.5 \mathrm{~dB}$. When we evaluate the interference from the SB-Tx, the SA-Rx for which the CINR ranges from $\gamma_{\text {req }}$ to $\alpha \gamma_{\text {req }}$ is not counted as an interfered receiver. Since $\alpha$ is changed, in order to make $\rho_{\max }$ of the IS and the CS equal to $1 \%, T_{\mathrm{IS}}, T_{\mathrm{CS}}$, and $T_{\mathrm{CSCINR}}$ are set to be $-109.9 \mathrm{dBm}$, $-90.2 \mathrm{dBm}$, and $-90.2 \mathrm{dBm}$, respectively. Figures 8 and 9 show the obtained results. If there is no margin in the received signal level, the only way to avoid the interference with the system A is stopping the transmission of the SBTx. When we assume a small margin in the received signal level, the CSCINR can reduce the interference to the SA-Rx completely under the assumption of this paper. In addition, when there is a small margin, the SB-Tx does not need to reduce the transmit power greatly. As a result, $p_{\text {link }}$ of the 


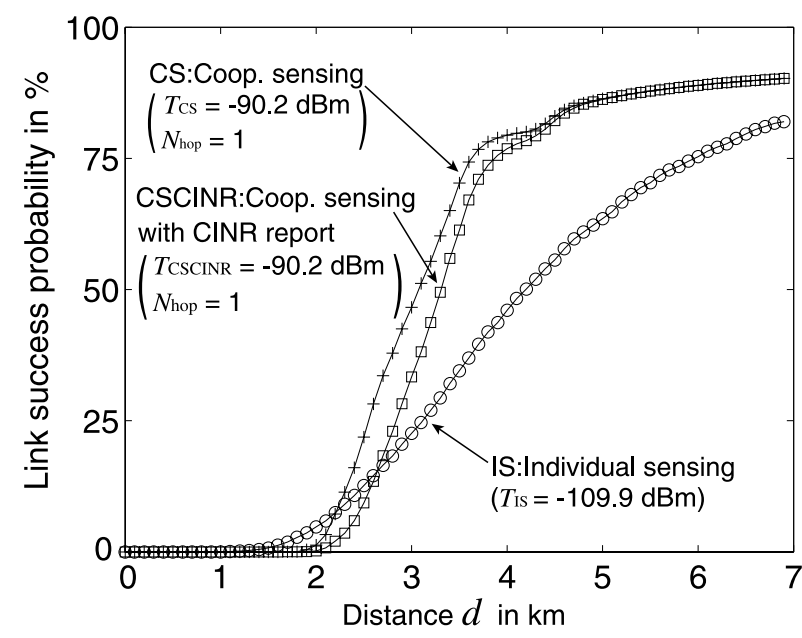

Fig. 9 Link success probability of system B $p_{\text {link. The receiver of system }}$ A has a margin of $0.5 \mathrm{~dB}$ or more in the received signal level.

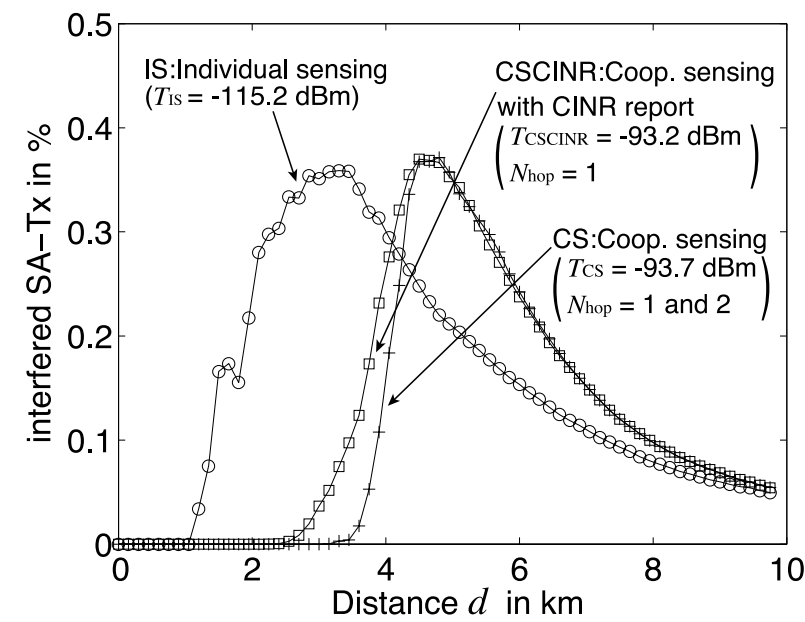

Fig. 10 Ratio of system A receivers interfered with by SB-Tx. $\rho_{\max }$ of all sensing schemes is fixed to $0.37 \%$.

CSCINR is improved significantly, as shown in Fig. 9.

\subsection{Improvement of System B Link Success Probability}

In this section, we investigate the link success probability of the CSCINR by fixing $\rho_{\max }$ of all sensing schemes at $0.37 \%$. The ratios of the SA-Rx that interfered with the SB-Tx are shown in Fig. 10. In order to reduce the interference to the system A, the detection probability of the IS and the CS are changed. $T_{\mathrm{IS}}$ is set to $-115.2 \mathrm{dBm}$, and $T_{\mathrm{CS}}$ is set to $-93.7 \mathrm{dBm}$. In the CS, the transmission of the SB-Tx is determined based on the sensing information from $N_{\mathrm{hop}, j}=1$ and $N_{\text {hop }, j}=2$, while $N_{\text {hop }, j}=1$ is employed in Sect. 3.2. The detection probability of the CSCINR remains equal to that in Sect. $3.2\left(T_{\mathrm{CSCINR}}\right.$ is set to $-93.2 \mathrm{dBm}$, and $N_{\mathrm{hop}, j}=1$ is employed in the decision of the transmission).

Figure 11 shows $p_{\text {link }}$ of three sensing schemes. In the IS and the CS, the suppression of the interference to the system A is realized simply by improving the detection proba-

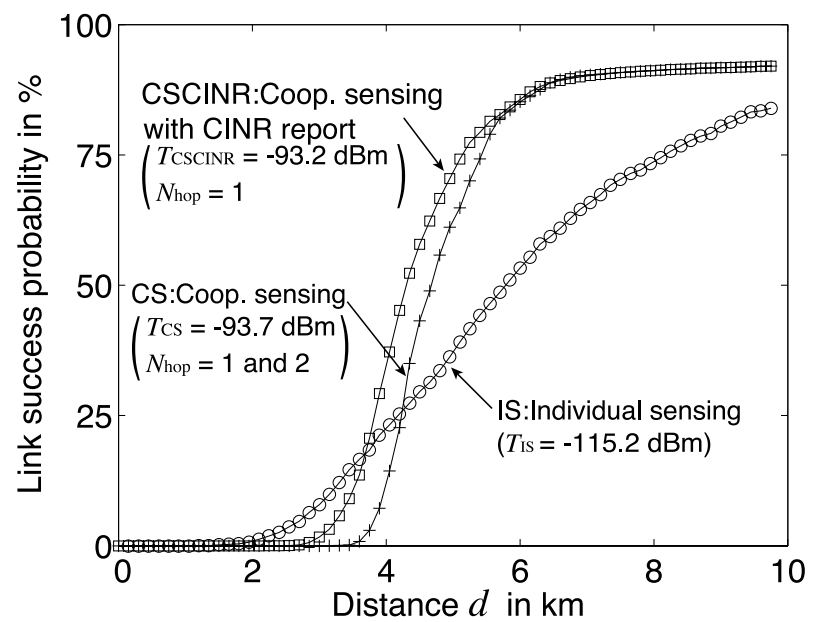

Fig. 11 Link success probability of system B $p_{\text {link }} \cdot \rho_{\max }$ of all sensing schemes is fixed to $0.37 \%$.

bility. As a result, although the SB-Tx is far from the system A service area, the transmission is suppressed. The false detection probability of the IS and the CS is increased. However, the CSCINR estimate the interference conditions of the system A and when the interference to the system A exceeds the permissible level, the transmit power is adjusted. Therefore, the CSCINR has a greater chance to transmit, and this leads to an improvement in $p_{\text {link }}$.

\section{Conclusions}

In this paper, we proposed a new cooperative sensing scheme for sharing the frequency band among multiple systems. In the proposed system, the interference conditions of high-priority system is estimated by the station of lowpriority system. Based on the estimated interference information, the transmit power is adjusted. The proposed system can reduce the interference to other systems more than the other sensing schemes, while having the same detection probability. Unfortunately, as a result of the shadowing effects, the interference to other systems cannot be reduced completely. However, if we consider the small margin in the received signal level, the proposed system can reduce the interference completely under the assumption of this paper. Moreover, the proposed system improves the link success probability of its system when the outage probability of another system is the same among other sensing schemes. Consequently, in order to improve the link success probability while maintaining the outage probability, it is more effective to estimate the interference conditions rather than make the detection probability extremely high.

\section{References}

[1] J. Mitora, III and G.Q. Maguire, Jr., "Cognitive radio: Making software radios more personal," IEEE Pers. Commun., vol.6, no.4, pp.13-18, Aug. 1999.

[2] S. Haykin, "Cognitive radio: Brain-empowered wireless communications,” IEEE J. Sel. Areas Commun., vol.23, no.2, pp.201-220, Feb. 2005. 
[3] D. Cabric, S.M. Mishra, D. Willkomm, R.W. Broderson, and A. Wolisz, "A cognitive radio approach for usage of virtual unlicensed spectrum," Proc. 14th IST Mobile Wireless Communications Summit 2005, June 2005.

[4] S.M. Mishra, A. Sahai, and R.W. Brodersen, "Cooperative sensing among cognitive radios,” Proc. ICC 2006, pp.1658-1663, Istanbul, June 2006.

[5] D. Cabric, S.M. Mishra, and R.W. Brodersen, "Implementation issues in spectrum sensing for cognitive radios," Proc. Asilomar Conference on Signals, Systems, and Computers, pp.772-776, 2004.

[6] M. Matsui, H. Shiba, K. Akabane, and K. Uehara, "Study of cooperative sensing for cognitive radio," IEICE Technical Report, SR200667, March 2007

[7] G. Ganesan and Y. Li, "Agility improvement through cooperative diversity in cognitive radio," Proc. IEEE Globecom, pp.2505-2509, St. Louis, Missouri, Nov.-Dec. 2005.

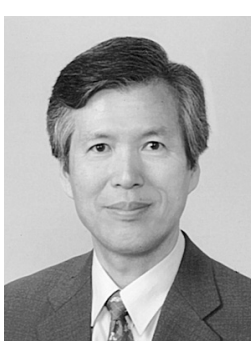

Susumu Yoshida received the B.E., M.E. and $\mathrm{Ph} . \mathrm{D}$. degrees in electrical engineering from Kyoto University, Kyoto, Japan in 1971, 1973 and 1978, respectively. Since 1973, he has been with the Faculty of Engineering, Kyoto University and currently he is a full professor of the Graduate School of Informatics, Kyoto University. During the last 30 years, he has been mainly engaged in the research of wireless personal communications. His current research interest includes highly spectrally efficient wireless transmission techniques and wireless ad hoc networks. During 19901991, he was a visiting scholar at WINLAB, Rutgers University, U.S.A. and Carleton University in Canada. He served as an Executive Committee Chair of PIMRC'99, Osaka and also as a Technical Program Committee Chair of IEEE VTC 2000-Spring, Tokyo. He was a guest editor of IEEE JSAC on Wireless Local Communications published in April and May 1996. He was a Director, Journal and Transactions of IEICE during 2002-2004, and is currently President of Communications Society of IEICE. He was awarded the Achievement Award in 1993 from the IEICE.

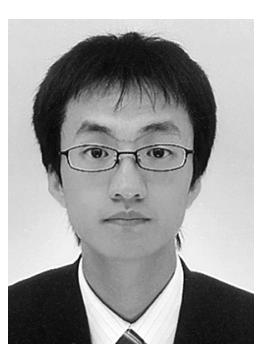

Youngjin Yu received a B.E. degree from the school of engineering science from Osaka University in 2006 with the support of a KoreaJapan joint scholarship for undergraduate students in science and engineering. He is currently studying towards his M.E. degree at the graduate school of informatics at Kyoto University. His current research interests include wireless communications systems with a particular emphasis on spectrum-sharing technology.

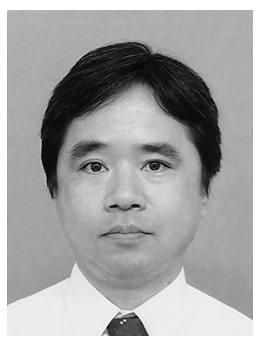

Hidekazu Murata received B.S., M.S., and $\mathrm{Ph} . \mathrm{D}$. degrees in electronic engineering from Kyoto University, Kyoto, Japan, in 1991, 1993 , and 2000, respectively. In 1993, he joined the Faculty of Engineering, Kyoto University. In 2002-2006, he was an associate professor of Tokyo Institute of Technology. Since 2006, he has been an associate professor of Kyoto University. His current research interests include signal processing and its hardware implementation, with particular application to multi-hop cooperative wireless networks. He received the Young Researcher's Award from the IEICE of Japan in 1997, the Ericsson Young Scientist Award in 2000, and the Young Scientists' Prize of the Commendation for Science and Technology by the Minister of Education, Culture, Sports, Science and Technology in 2006. He is a member of the IEEE and SITA.

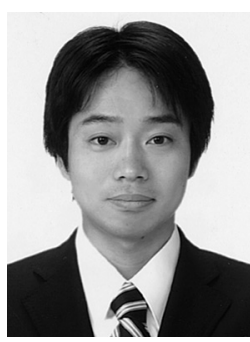

Koji Yamamoto received the B.E. degree in electrical and electronic engineering from Kyoto University in 2002, and the M.E. and Ph.D. degrees in informatics from Kyoto University in 2004 and 2005, respectively. Since 2005, he has been an assistant professor of the Graduate School of Informatics, Kyoto University. His research interests include multi-hop radio networks and distributed controls. He received the PIMRC 2004 Best Student Paper Award in 2004 and the Ericsson Young Scientist Award in 2006.

He is a member of the IEEE. 